\title{
Perfil Obstétrico de Adolescentes Embarazadas atendidas en un Hospital Público de la Ciudad de México."
}

\section{Obstetric Profile of Pregnant Adolescents in a Public Hospital in Mexico City.}

Hugo Tapia Martínez•

Araceli Jiménez Mendoza.•

Iñiga Pérez Cabrera...

\section{Resumen}

Introducción: A pesar de que se han realizado esfuerzos para disminuir el embarazo en la adolescencia y con ello sus daños a la salud, aún persiste como problema de salud en el mundo y en el país. Este artículo corresponde a la primera etapa de una investigación más amplia. Responde al Objetivo específico de caracterizar el perfil obstétrico de adolescentes gestantes que acuden a su vigilancia prenatal en un Hospital de la Ciudad de México. El perfil abarca factores de riesgo biológico, psicológico y social.

Metodología: Estudio descriptivo transversal realizado en el periodo de septiembre-noviembre de 2011, en una muestra por conveniencia de 100 adolescentes que se encontraban en la segunda mitad del embarazo. Instrumento Previgen II y III del SERP CIMIGen (1995).

"PAPIIT IN307811-3, ENEO-UNAM 054. GRUPO DE INVESTIGACIÓN EN ESTUDIOS DE GÉNERO Y SALUD DE LAS MUJERES

-Maestro en Ciencias de la Enfermería. Profesor de Carrera Asociado B Tiempo Completo ENEO-UNAM - Maestra en Ciencias de la Enfermería. Profesor de Carrera Titular A Tiempo Completo ENEO-UNAM ...Maestra Decana ENEO-UNAM

Fecha de ReCibido: 4 De Febrero 2012

Fecha de enviado: 22 De Febrero 2012

FECHA DE Aprobado: 18 DE MAYO 2012

Palabras

Clave:

Adolescencia, embarazo, factores de riesgo 
Resultados: 96\% tuvo riesgo medio para la edad (15 a 19 años); aunque 9 tuvieron mayor riesgo debido a tenían 15 años o menos; $27 \%$ eran solteras,23\%correspondían a un nivel socioeconómico bajo, dos terceras partes reportó una escolaridad de 9 años o menos. Respecto a los factores biológicos cuatro de cada 10 tuvieron riesgo medio o alto en función de su peso (35\% entre 41 a 50 kgs. y $6.1 \%$ con menos de 40 kgs); la gran mayoría eran nuliparas; $12 \%$ con tabaquismo positivo; $16 \%$ tuvo amenaza de aborto; $11 \%$ con amenaza de parto pretermino; el problema de salud más importante fue la infección de vías urinarias $28 \%$ la tenía controlada y $10 \%$ activa.

Discusión y Conclusión: Los resultados coinciden con otras investigaciones, pero el número de embarazadas de 15 años o menos es superior a la media nacional que es de 6 en mil; el perfil obstétrico es en mayor medida de mediano riesgo; falta conocer los riesgos y daños en el posparto y recién nacido para completar este perfil.

Key Words: adolescence, pregnancy, riskfactors.

\section{INTRODUCCIÓN}

El embarazo en la adolescencia continua siendo un problema de salud pública a nivel global, se estima que cada año 14 millones de adolescentes (mujeres de entre 10 y 19 años) dan a luz en el mundo, lo cual representa poco más del $10 \%$ del total de nacimientos. El $80 \%$ de los nacimientos tienen lugar en los países en desarrollo. ${ }^{1}$ Para el 2010, de acuerdo

\section{Abstrac}

Introduction: Despite the efforts to decrease its prevalence and consequences, pregnancy in adolescents is still a health problem worldwide. This study represents the first stage of a wider research on the issue.

objective: To characterize the obstetric profile of pregnant adolescents who attend a public hospital in Mexico City for prenatal monitoring. The profile includes biological, psychological and social risk factors

Methodology: Basic descriptive study developed in the period of September-November 2011 from a sample of 100 adolescents at the second half of their pregnancies. The instruments used were the Previgen II and III of SERP CIMIGen (1995)

Results: 96\% were at moderate risk for the $15-19$ years old age group. $27 \%$ were single. $23 \%$ were in the low socioeconomic level. Two thirds reported 9 or less years of education. Four out of ten were at moderate or high risk in relation to their weight (35\% weighted between 41 and 50 kilograms, and $6.1 \%$ weighted 40 or less kilograms). The majority had never delivered before $12 \%$ were tobacco users, $16 \%$ were at risk of abortion, and $11 \%$ were at risk of premature delivery. Urinary infection was an important problem, and while $28 \%$ were controlled, $10 \%$ were not.

Discussion and Conclusion: These results are similar to those of other studies. The overall obstetric profile is characterized by having a moderate risk, but it is necessary to gather information on the risks and damages after delivery as well as on the baby in order to complement this profile. 
La tasa de embarazo en las adolescentes de 12 a 19 años de edad fue de 79 por cada mil mujeres. ${ }^{3}$ Una de cada seis mujeres que se embarazan es adolescente. La mortalidad por embarazo en la adolescencia, está relacionada con una mayor mortalidad materna; ${ }^{4}$ el riesgo de muerte materna en adolescentes es el doble que en el resto de las mujeres de edad fértil, y cuatro veces mayor cuando se trata de menores de 15 años. ${ }^{5}$

En el rubro de morbilidad, las adolescentes embarazadas constituyen un grupo de riesgo, ya que están más expuestas a complicaciones como la eclampsia, anemia, parto prematuro y a tener recién nacidos de bajo peso, ${ }^{6}$ complicaciones relacionadas con factores de riesgo como la pobreza, desnutrición, escolaridad limitada y falta de apoyo familiar ${ }^{5}$, que a su vez, provocan, la ausencia o asistencia tardía al control prenatal y consecuentemente a un déficit de información y orientación sobre las recomendaciones y cuidados durante el embarazo y parto.

Los factores de riesgo (FR) se entienden como aquellas características o atributos que aumentan la probabilidad de que ocurra algún daño a la salud de las personas. ${ }^{7}$ Por ejemplo, el factor de riesgo tabaquismo positivo, implica que las mujeres que fuman en el embarazo tienen más probabilidades de que sus hijos tengan bajo peso al nacer en comparación, con aquellas mujeres que no fuman. En el terreno de la Obstetricia los FR representan las características que se presentan en el transcurso del embarazo, parto, puerperio y recién nacido; para lo cual, los expertos han realizado distintas clasificaciones que van desde números, colores e incluso software, con el fin de sistematizarlos y con ello realizar medidas anticipatorias para cancelar, disminuir o modificar el o los factores de riesgo y evitar daños a la salud materna. Asimismo, los FR han sido clasificados en biológicos, psicológicos y sociales. Entre los FR biológicos se encuentran por ejemplo: la edad, el peso, la talla o el número de embarazos; mientras que en los de orden social se hallan: el nivel socioeconómico, el estado civil o la escolaridad; y dentro de los psicológicos estarían: la actitud de la mujer ante el embarazo, el nivel de depresión o el estrés.

La adolescencia es una de las etapas de vida del ser humano, caracterizada por cambios físicos, psicológicos, sociales y culturales; en la que las personas transitan de una inmadurez a una madurez como adultos. De ahí que la Organización Mundial de la Salud, la define como el período de la vida en el cual el individuo adquiere madurez reproductiva, transita los patrones psicológicos de la niñez a la adultez y establece su independencia socioeconómica, ${ }^{8}$ ya que están presentes muchas de las características de la etapa anterior junto con otras nuevas. Para fines prácticos abarca de los 10 a los 19 años y se divide en adolescencia temprana y adolescencia tardía, de los 10 a 14 y de los 15 a 19 años respectivamente. $^{3}$

En esta etapa muchos(as) adolescentes, experimentan distanciamiento con sus progenitores y un mayor acercamiento con sus amistades, quienes en un buen porcentaje, suplen las necesidades de información. Aumentando la situación de vulnerabilidad, a problemas de salud como el embarazo no pla- neado, tienen menos información y preparación para las prácticas de cuidado durante su embarazo como la estimulación prenatal, higiene y cuidados personales, alimentación y sustancias toxicas, en comparación con las mujeres gestantes adultas. ${ }^{9}$

Por consiguiente, tienen más riesgos y complicaciones en su salud durante el embarazo y parto así como la de sus hijos que las mujeres de 20 años o más. ${ }^{10}$ Por ello, Identificar los factores de riesgo de las adolescentes embarazadas pertenecientes a determinados sectores poblacionales puede favorecer realizar intervenciones fundamentadas acordes a sus necesidades específicas.

\section{Objetivos}

Caracterizar el perfil obstétrico de las adolescentes gestantes que acuden al control prenatal en un Hospital Público de la Ciudad de México.

\section{Metodología}

Se trata de un estudio observacional, descriptivo y transversal, la muestra la constituyeron 100 adolescentes embarazadas elegidas por conveniencia, ellas fueron referidas de dos centros de salud al Hospital General Iztapalapa (HGRI) de la Secretaría de Salud del Distrito Federal Ciudad de México durante los meses de septiembre a noviembre de 2011, los criterios de inclusión fueron: ser adolescentes gestantes en la segunda mitad del embarazo; llevar control prenatal subsecuente; sin trabajo de parto y que desearan participar en el estudio.

Los instrumentos utilizados corresponden a las Cédulas de riesgo perinatal llamadas "Previgen" II y III del Sistema de Evaluación de Riesgo Reproductivo y Perinatal (SERRP) propuestas por el Centro 
de Investigación Materno Infantil del Grupo de Estudios al Nacimiento (CIMI Gen) y la Escuela Nacional de Enfermería y Obstetricia (ENEO) en 1995; que permiten la evaluación efectiva de los factores de riesgo en la etapa reproductiva y perinatal; para su eliminación, modificación o disminución de su capacidad de afectar la salud materna y referir oportunamente los casos de alto riesgo al nivel subsecuente de atención. Se sustenta en la semaforización, color verde indica Riesgo Bajo (RB); amarillo Riesgo Medio (RM) y; rojo Riesgo Alto (RA), es decir, en una escala ordinal. La primera parte mide el riesgo al inicio del embarazo y la segunda parte durante el mismo. El instrumento ha sido utilizado en el CIMIGEN por más de 15 años para brindar atención materno/infantil por enfermeras capacitadas. Consta de 54 factores de riesgo a los cuales se sumaron 10 reactivos sociodemográficos, quedando un total de 62 .

Seis becarios del pregrado de la Licenciatura en Enfermería y Obstetricia previamente capacitadas sobre los antecedentes y objetivos del proyecto, así como del manejo del instrumento y las instrucciones para la aplicación, llevaron a cabo la recolección de los datos.

Los datos se transfirieron a una base de datos y se codificaron por medio del programa spss versión 15 , que sirvió para la presentación de los cuadros y su análisis.

La investigación atendió los principios éticos de beneficencia y no maleficencia, veracidad, privacidad y confidencialidad, señalados en el código ético de Enfermería. ${ }^{11}$ Por otro, su ejecución se sustentó en el marco de lo señalado en el Reglamento de la Ley General de Sa- lud en los artículos 34, 36, 38 y 39 que aluden al riesgo mínimo para los sujetos de estudio, al consentimiento informado, al anonimato y a la libertad de retirarse en cualquier momento del estudio. ${ }^{12}$

Se obtuvo el consentimiento informado de las adolescentes gestantes junto con la persona responsable (madre, padre, tutor o pareja) antes de llevar a cabo las entrevistas (tanto para las entrevistas directas como para el llenado de los instrumentos diseñados). Se brindó una explicación de los objetivos del estudio y se les describió claramente en lo que consistía su participación en el estudio. Posteriormente se dio lectura conjuntamente con la paciente al formato para consentimiento informado, proporcionándole las facilidades para expresar sus dudas y aclarárselas. Se hizo énfasis en que su participación era completamente voluntaria, que el empleo de la información era completamente confidencial y que en caso de negativa a participar no modificaría en lo absoluto la atención que le proporciona el Hospital. Cuando aceptaron participar, firmaron el formato.

\section{Resultados}

Completaron la encuesta 99 adolescentes, la obtención de los datos fue entre la semana 30 y 36.6 de embarazo. En cuanto a los antecedentes perinatales se muestran los siguientes resultados. Tabla No. 1.

En este grupo de estudio cuatro adolescentes quedaron clasificadas en riesgo alto en función de que son menores de 14 años; cinco de 15 años; veinte de 16 años; diecisiete de 17 años; veintinueve de 18 años y; veinticuatro de 19 años.

La clasificación de riesgo en función de las variables sociodemográ- ficas de este grupo de adolescentes muestra que: una tercera parte es de riesgo medio en virtud de que son solteras; de acuerdo con la escolaridad dos terceras partes corresponden a riesgo alto o medio ya que sólo tenían estudios de primaria o secundaria respectivamente; al cuestionarlas sobre a cual nivel socioeconómico pertenecían, una cuarta parte se ubicaron en riesgo alto en función de que refirieron el nivel muy bajo y el resto de las adolescentes en riesgo medio pues se ubicaron en el nivel socioeconómico medio y; al preguntarles ¿cómo se siente con su embarazo? bien, regular o mal,noventa y siete adolescentes se clasificaron con una actitud adecuada (bien) hacia su embarazo, lo que las enmarcó en riesgo bajo. En conjunto estas características (estado civil, escolaridad y nivel socioeconómico) reflejan carencias importantes y son indicadores de las condiciones de la zona en que está ubicado el Hospital y de sus habitantes.

Las variables perinatales que contribuyeron para clasificar a las adolescentes en riesgo alto fueron el bajo peso al inicio del embarazo $(<40 \mathrm{Kg})$ y la consanguinidad; en cambio aquellas variables que en mayor medida aportaron para que fueran clasificadas en riesgo medio fueron nuevamente el peso (de 41 a $50 \mathrm{Kg}$.), el ser en su mayoría nulíparas (88\%), aunque 6\% tenían el antecedente de una cesárea previa $\mathrm{y}$ un nivel de hemoglobina entre $8 \mathrm{y}$ 10.9; otras variables que contribuyeron para este riesgo medio fueron las toxicomanías (tabaquismo, alcoholismo y drogadicción con 12 , 7 y $3 \%$ respectivamente).

Los factores de riesgo en el embarazo actual se muestran en la Tabla No. 2 


\section{Tabla 1 Perfil Antecedentes Perinatales*}

\begin{tabular}{|c|c|c|c|c|c|c|}
\hline Factor de Riesgo & Riesgo Bajo & $\%$ & Riesgo Medio & $\%$ & Riesgo Alto & $\%$ \\
\hline Edad & & & 15 a 19 & 96 & 14 ó menos & 4 \\
\hline \multicolumn{7}{|c|}{ Variables sociodemográficas } \\
\hline Edo. Civil & Unión libre & 60 & Solteras & 27 & \multirow{3}{*}{6 ó menos } & \multirow{3}{*}{11} \\
\hline Escolaridad & 10 y más & 33 & 7 a 9 & 55 & & \\
\hline Ocupación & Hogar & 88 & & & & \\
\hline Nivel Socioeconómico & Alto y Medio & 1 & Medio bajo & 72 & \multirow[t]{2}{*}{ Muy Bajo } & \multirow[t]{2}{*}{23} \\
\hline Actitud & Adecuada & 99 & Inadecuada & 1 & & \\
\hline \multicolumn{7}{|l|}{ Variables perinatales } \\
\hline Peso al inicio & 51 a $60 \mathrm{Kg}$. & 49 & 41 a $50 \mathrm{Kg}$. & 35 & \multirow[t]{7}{*}{40 ó menos } & \multirow[t]{7}{*}{6} \\
\hline Paridad & 1 a 3 partos & 11 & Nulíparas & 89 & & \\
\hline Cesáreas previas & No & 93 & Una & 6 & & \\
\hline Parto pretérmino & No & 98 & Uno & 1 & & \\
\hline Hijos pequeños & No & 96 & Uno & 3 & & \\
\hline Hijos grandes & No & 98 & Uno & 1 & & \\
\hline Muerte perinatal & No & 98 & Uno & 1 & & \\
\hline Consanguinidad & No & 93 & & & \multirow[t]{5}{*}{$\mathrm{Si}$} & \multirow[t]{5}{*}{2} \\
\hline Hemoglobina & 11 y más & 71 & 8 a 10.9 & 6 & & \\
\hline Tabaquismo & No & 87 & Sí & 12 & & \\
\hline Alcoholismo & No & 92 & Sí & 7 & & \\
\hline Drogadicción & No & 97 & Sí & 3 & & \\
\hline
\end{tabular}

\section{Tabla 2 Perfil Factores de Riesgo en el Embarazo*}

\begin{tabular}{|c|c|c|c|c|c|c|}
\hline Factor de Riesgo & Riesgo bajo & $\%$ & Riesgo medio & $\%$ & Riesgo alto & $\%$ \\
\hline Amenaza de aborto & No & 82 & Controlada & 16 & \multirow{5}{*}{ Activa } & \multirow{5}{*}{4} \\
\hline $\mathrm{APP}$ & No & 83 & Controlada & 11 & & \\
\hline Nefropatías & No & 98 & Controlada & 1 & & \\
\hline Diabetes & No & 97 & Controlada & 2 & & \\
\hline HIE & No & 98 & Controlada & 1 & & \\
\hline IMC & $\begin{array}{l}\text { Normal (18.5- } \\
\text { 24.9) }\end{array}$ & 68 & $\begin{array}{l}\text { Sobrepeso } \\
(25-29.9) \\
\text { Infrapeso } \\
\text { (menos de 18.5) }\end{array}$ & 17 & $\begin{array}{l}\text { Obesidad } \\
\text { (30 y más) }\end{array}$ & 1 \\
\hline \multirow[t]{2}{*}{ Aumento de peso por mes } & \multirow[t]{2}{*}{$1-1.5 \mathrm{Kgs}}$. & \multirow[t]{2}{*}{54} & 1.6 a $2.9 \mathrm{Kg}$. & 26 & Más de $3 \mathrm{Kg}$. & 7 \\
\hline & & & Sin aumento & 4 & Disminución & 4 \\
\hline $\begin{array}{l}\text { Contracciones antes } \\
\text { de la semana } 37\end{array}$ & 1 y menos & 92 & $\begin{array}{l}2 \text { a } 3 \text { contracciones } \\
\text { en } 10 \mathrm{~min} .\end{array}$ & 5 & & \\
\hline Infección de Vías Urinarias & No & 58 & Controlada & 28 & Activa & 10 \\
\hline
\end{tabular}


Como se muestra en esta tabla la infección de vías urinarias fue el factor de riesgo más frecuente en el embarazo actual del grupo estudiado; afortunadamente 3 de cada cuatro tenían el problema controlado por lo que se ubicaron en riesgo medio y sólo en una de cada cuatro mujeres la infección estaba activa,conforme a esto se encontraban en riesgo alto.

En función de su frecuencia otras situaciones que contribuyeron para ubicar a las mujeres en riesgo medio o alto fueron el aumento mensual de peso ya sea por un aumento mayor al establecido (33 casos) o la situación contraria por no aumento (4 casos) o inclusive disminución (4 casos). En estrecha relación con este factor se encuentra la clasificación según el Índice de Masa Corporal (IMC) que las mujeres tenían al inicio del embarazo conforme al cual se clasificaron con obesidad, sobrepeso, normal o infrapeso; las situaciones diferentes de un estado de nutrición normal llevan a considerarlas en riesgo alto, o medio ( $25 \mathrm{y}$ un caso respectivamente).

Por otra parte refirieron haber padecido amenaza de aborto (16 casos) y amenaza de parto pretermino (15 casos, en 11 ya estaba controlada, pero cuatro se ubicaban en riesgo alto pues se mantenía activa). En relación a la presencia de contracciones uterinas antes del embarazo de término, cinco estuvieron en riesgo medio en función de que presentaron de 2 a 3 contracciones uterinas en diez minutos.

Finalmente, en enfermedades crónicas asociadas con el embarazo, cuatro estuvieron en riesgo medio en virtud de que una refirió nefropatía; dos diabetes y otra con hipertensión inducida por el embarazo.

\section{Dıscusıón}

El estudio tuvo como objetivo caracterizar el perfil obstétrico de las adolescentes gestantes que acuden al control prenatal en un Hospital Público de la Ciudad de México ubicado en una de sus delegaciones más densamente pobladas y entre las tres que tienen mayores porcentajes de pobreza patrimonial. ${ }^{13}$

Los resultados obtenidos muestran que la mayoría de adolescentes embarazadas tuvieron una edad entre 16 a 19 años; sin embargo 9 se embarazaron a los 15 años o menos, esto expresado como razón es nueve de noventa y nueve, dato muy elevado comparado con la cifra a nivel nacional en 2011, en que hubo una tasa de embarazo en las adolescentes de 12 a 15 años de seis por cada mil. ${ }^{3}$

De acuerdo con su edad la mayoría de estas adolescentes deberían haber iniciado o estar estudiando el bachillerato sin embargo refirieron estudios de 9 años o menos (primaria y secundaria), y se dedican al hogar; es decir ya habían suspendido sus estudios,situaciones similares se han observado en otros estudios en población latinoamericana ${ }^{6,14,15}$. Pareciera, conforme a esto, que los factores asociados al embarazo podrían estar persistiendo, si se toma en cuenta que el fenómeno del embarazo en la adolescencia es multifactorial, con más frecuencia en los sectores desprotegidos, como las comunidades de la Delegación de Iztapalapa, en donde la unión y maternidad tempranas, aún constituyen formas de vida femeninas, limitadas por las estructuras sociales y culturales. Por lo cual, una mayor información y acceso a metodologías anticonceptivas probablemente tendrá poca efectividad para pre- venir el embarazo, ya que no existe la motivación ni las condiciones para postergar la maternidad. ${ }^{14}$

La mayoría de las adolescentes gestantes acudieron al Hospital General Regional Iztapalapa referidas de Centros de Salud, al final del embarazo de 30 a 36.6 semanas de gestación sin su pareja. Es sabido que las adolescentes al enterarse del embarazo, entran en un proceso de crisis por el impacto psicosocial en sus vidas en lo individual, familiar, escolar o laboral, ${ }^{16}$ aunado a los cambios psicosociales propios de la adolescencia, razón por la cual sus parejas sexuales evaden la responsabilidad ${ }^{17} y$ varias de ellas acuden acompañadas de otras personas; asimismo, cuando el crecimiento uterino se hace evidente, más de 20 semanas de gestación e incluso más tarde o al manifestar trabajo de parto, es cuando asisten por primera vez a la consulta prenatal, lo cual las convierte en mujeres con embarazo de alto riesgo.

Casi la mitad respondió un peso al inicio del embarazo de 41 a 50 kgs., esto puede responder a la interrelación con otros factores de riesgo como el bajo nivel socioeconómico y la baja escolaridad de la mayoría de las encuestadas, ello se constata con la presencia de $6 \mathrm{ca}$ sos con baja hemoglobina, lo cual es común ver en las mujeres que viven en las comunidades de Iztapalapa; como lo afirman los autores: son situaciones ligadas a la pobreza y deficiencias alimenticias. ${ }^{3}$ En este mismo tenor de interrelación de factores de riesgo, se tiene la presencia de las Toxicomanías en el grupo de estudio, ya que 22 de ellas mencionaron haberlas probado, de mayor prevalencia el tabaco, le sigue el alcohol y otras drogas; 
estas características coinciden con lo reportado a nivel nacional para el grupo de adolescentes: $41.9 \%$ de las mujeres adolescentes han fumado alguna vez, $7.1 \%$ de mujeres adolescentes han consumido alcohol en grandes cantidades en alguna ocasión y el 1.9\% de las adolescentes ha consumido alguna otra droga. ${ }^{18}$

Las características o atributos que aumentan la probabilidad de que ocurra algún daño a la salud de las personas, que constituyen el concepto de factor de riesgo, se retoma en esta discusión, considerando que el factor más significativo fue la infección de vías urinarias, el cual se presentó en más de un tercio; los daños asociados a este factor fueron la amenaza de aborto que se presentó en 16 casos y la amenaza de parto pretermino en 11 casos, incluyendo 5 casos con presencia de contracciones uterinas; ello concuerda con la literatura; la infección de vías urinarias, afecta al 6-12\% de todas las gestaciones, en sus diferentes presentaciones como bacteriuria asintomática, cistitis y pielonefritis y las bacterias que intervienen, comúnmente ocasionan actividad uterina. ${ }^{19,20}$

El bajo peso al nacer o el Apgar bajo, relacionados con estos factores no se han medido porque las adolescentes aún se encontraban embarazadas.

\section{Conclusiones}

Se obtuvo un perfil obstétrico de adolescentes embarazadas de comunidades desprotegidas de la Delegación Iztapalapa Distrito Federal México.

El estudio mostró que las adolescentes se embarazan principalmente entre los 16 y los 19 años; sin embargo, hubo un incremento de embarazos en las adolescentes de 15 años y menos edad, la cifra nacional es de 6 en mil contra nueve en cien adolescentes en esta investigación.

Se dedican al hogar; pertenecen al Distrito Federal; más de la mitad tienen sólo estudios de primaria y secundaria; asisten al control prenatal sin su pareja. Casi la mitad tuvo un peso al inicio entre 40 o menos Kg.y de 41 a 50 kg. Predomina el nivel socioeconómico bajo; la mayoría son nulíparas y refieren una buena actitud ante el embarazo; hubo amenaza de aborto en 16 casos; 15 con amenaza de parto pretermino; y más de un tercio refirió infección de vías urinarias. Con ello se tiene un perfil de los riesgos que tienen las adolescentes embarazadas de nivel socioeconómico bajo, que coincide con la literatura.

Por lo anterior se concluye que el perfil obstétrico es de mediano y alto riesgo en las adolescentes estudiadas; queda pendiente completar el perfil obstétrico en el parto, posparto y recién nacido, que se evaluarán una vez que se establezcan estas etapas en las mujeres, con la utilización de las cédulas correspondientes. No obstante, se sugiere que las instancias de salud y educativas, realicen acciones para prevenir y controlar oportunamente la infección urinaria como factor de riesgo frecuente en este estudio.

\section{Agradecimientos}

Al Hospital General Regional Iztapalapa por facilitar las instalaciones para llevar a cabo este estudio.

A las Enfermeras del Servicio de Control Prenatal del HGR Iztapalapa.

\section{Referencias Bibliograficas}

1 UNFPA. The State of World Population 2004: The Cairo Consensus at Ten: Population, Reproductive Health and the Global Effort to End Poverty, p. 76. New York: UNFPA. 2004. [Consultado 20 febrero 2010] Disponible en: http://bit.ly/ $\mathrm{xV} 4 \mathrm{qcA}$

2 INEGI. Censo General de Población y Vivienda 2010: Tabulados del Cuestionario Básico. México, 2011.

3 Secretaria de Salud. Perfil epidemiológico de la población adolescente. SSA. Subsecretaria de Prevención y Programación Dirección General de Epidemiología. México 2011.

4. Organización Panamericana de la Salud. IMAN Servicios: Normas de atención de salud sexual y reproductiva de adolescentes. Washington, D.C. OPS, 2005. [Consultado 13 Enero 2010] Disponible en:http://bit.ly/AenkWA

5 Ximenes-Neto FRG, Marques MS, Rocha J. Problemas Vividos por las Adolescentes durante la Gestación. Revista Electrónica Enfermería Global. 2008. No. 12 Febrero. [Consultado 06 Marzo 2010] Disponible en: http://bit.ly/wd8L4P

6 Secretaría de Salud. Género y Salud en Cifras. Boletín Vol.6 No.1 Enero-Abril 2008. [Consultado $19 \mathrm{Fe}-$ brero 2010] Disponible en: http:// bit.ly/Atjjjw

7 Centro Latinoamericano de Perinatología y Desarrollo Humano (CLAP)-OPS/OMS. Enfoque de Riesgo. Manual de Autoinstrucción. Montevideo Uruguay. [Consultado 11 Enero 2012] Disponible en: http://bit.ly/Av57fH

8 Risueño A, Motta I. La adolescencia existe o existen adolescentes: la adolescencia como constructo. Universidad Argentina John 


\section{Enfermería Universitaria}

F. Kennedy. 2008. [Consultado 20

Marzo 2010] Disponible en: http:// bit.ly/xQRmqp

9 Arévalo Sánchez E.Gestación y prácticas de cuidado. Avances en Enfermería. 2007; 25(2). [Consultado 10 Febrero 2010].En: http://bit.ly/ wATCoD

10 Valdés Dacal S, Essien J, Bardales Mitac J. Et al. Embarazo en la Adolescencia Incidencia, Riesgos y Complicaciones. Rev Cubana Obstet Ginecol 2002;28(2):84-8. [Consultado 17 marzo 2010].Disponible en: http://bit.ly/xUyWWf

11 Comisión Interinstitucional de Enfermería. Código de ética para las enfermeras y enfermeros de México. México: Secretaría de SaludIMSS-ISSSTE, 2001. Disponible en: http://bit.ly/RKTtTF

12 DOF. Reglamento de la Ley General de Salud en materia de investigación para la salud, Capítulo I, Titulo Segundo. De los aspectos éticos de la Investigación. México, 1986 [citado en mayo 2012]. Disponible en: http://www.salud.gob.mx

13 Consejo Nacional de Evaluación de la Política de Desarrollo Social. Mapas de pobreza y rezago social en 2005, Distrito Federal. México: CONEVAL 2005. Disponible en: http://bit.ly/QKfff3

14 Stern C. El embarazo en la adolescencia como problema público: una visión crítica. Salud Pública de México 1997; 39 (2): 137-143.

15 Fernández-Rodríguez Y, EscalonaAguilera JR. Comportamiento del embarazo en la adolescencia. Policlínico "José Marti”, Gibara. Junio 2009. Rev. Ped. Elec. [en línea] 2011, Vol 8, N 1. ISSN 0718-0918. [Consultado 10 febrero 2011]. Disponible en: http://bit.ly/yXFD5C

16 Ulanowicz MG, Parra KE, Wendler GE, Monzón LT. Riesgos en el Embarazo Adolescente. Revista de Posgrado de la VIa Cátedra de Medicina - N 153 - Enero 2006 13. [Consultado 4 febrero
2011] Disponible en: http://bit.ly/ wcfjNd

17 Secretaría de Salud. Prevención del embarazo no planeado en los adolescentes. México DF: SSA. Dirección General de Salud Reproductiva. Segunda Edición. 2002.

18 Consejo Nacional contra las Adicciones, Encuesta Nacional de Adicciones 2008. México, 2010. [Consultado 24 marzo 2012]. Disponible en: http://bitly.com/Uli$\mathrm{pHf}$

19 Quiroga C. Infección de Vías Urinarias en la Mujer Gestante. En: Elizalde R, Nassif JC, Borgi M, Savransky R. (Coord) Programa de Actualización en Gineco Obstetricia. México: Editorial Panamericana, 2000, Cuarto Ciclo, Módulo 1, Pag. 74-97.

20 Cunningham G, Leveno K, Bloom S, Hauth J, Gilstrap L, Wenstrom K. Obstetricia de Williams, 22a. ed. México: Editorial McGraw Hill, 2006. pp. 1095-1099. 